\title{
Préparation et études vibrationnelle et électronique de complexes de la phtalocyanine d'or avec des ligands axiaux pyridine et pyridines substituées
}

\author{
Ali Sanda BAWA ${ }^{1 *}$, Mabinty Bayo-BANGOURA ${ }^{2}$, Bertrand OUEMEGA $^{2}$ et \\ Karifa $\mathrm{BAYO}^{2}$ \\ ${ }^{1}$ Université d'Agadez, BP 199, Agadez, Niger. \\ ${ }^{2}$ Laboratoire de Chimie Moléculaire et des Matériaux ; Equipe Chimie de Coordination, Université de \\ Ouagadougou. 03 BP 7021 Ouagadougou 03, Burkina Faso. \\ *Auteur correspondant ; E-mail : sandabawaali@yahoo.fr; Tel: +226 74390447
}

\section{RESUME}

Les phtalocyanines de nombreux éléments périodiques ont été préparées et étudiées. Toutefois, il existe très peu de données disponibles sur la phtalocyanine d'or. Ce travail a pour objectif d'apporter une contribution à une meilleure connaissance de la structure électronique de ce complexe. La phtalocyanine d'or ( $\mathrm{AuPc}$ ) et une série de ses complexes portant des ligands axiaux pyridine (Py) et pyridines subtituées (R-Py) ont été préparées et étudiées par spectrométries vibrationnelle et optique. Les ligands utilisés sont: $3 \mathrm{MePy}$, 4MePy, 3HOPy, 4HOPy, 3ClPy, 4ClPy, $3 \mathrm{COHPy}, 4 \mathrm{COHPy}, 3 \mathrm{CNPy}, 4 \mathrm{CNPy}, 3 \mathrm{HOCH}_{2} \mathrm{Py}$ et $4 \mathrm{HOCH}_{2} \mathrm{CH}_{2} \mathrm{Py}$. L'analyse des spectres infrarouges a permis de mettre en évidence, dans les spectres des complexes, de nouvelles bandes de vibration attribuables aux ligands axiaux. En spectrométrie optique, une nouvelle bande de transition, absente dans le spectre de AuPc, apparaît autour de $575 \mathrm{~nm}$ dans les spectres des complexes. Elle est attribuable à une transition avec transfert de charges du métal central vers le ligand axial (TC $\mathrm{M} \rightarrow \mathrm{L}_{\mathrm{ax}}$ ). Nos résultats confirment que les transitions avec transfert de charges du ligand axial vers le macrocycle ( $\mathrm{TC} \mathrm{L}_{\mathrm{ax}} \rightarrow \mathrm{Pc}$ ) se font avec l'assistance des orbitales « $\mathrm{d}$ » du métal central ; elles ne sont possibles que lorsque la structure électronique de ces dernières le permette.

(C) 2018 International Formulae Group. All rights reserved.

Mots clés: Phtalocyanine d'or (AuPc), pyridine, pyridines substituées, spectrométrie vibrationnelle, spectrométrie optique.

\section{Preparation and study by vibrational and electronic spectrometries of a series of goldphthalocyanine (AuPc) complexes with pyridine Py and substituted pyridine R-Py axial ligands}

\begin{abstract}
The phthalocyanines of many periodic elements have been prepared and studied. However, there is very little information on gold phthalocyanine. The aim of this work is to contribute to a better knowledge of the electronic structure of this complex. Gold phthalocyanine (AuPc) and a series of its complexes with pyridine Py and substituted pyridines R-Py axial ligands were prepared and studied by vibrational and optical spectrometry. The ligands used are: $3 \mathrm{MePy}, 4 \mathrm{MePy}, 3 \mathrm{HOPy}$, $4 \mathrm{HOPy}, 3 \mathrm{ClPy}, 4 \mathrm{ClPy}, 3 \mathrm{COHPy}, 4 \mathrm{COHPy}, 3 \mathrm{CNPy}, 4 \mathrm{CNPy}, 3 \mathrm{HOCH}_{2} \mathrm{Py}$ and $4 \mathrm{HOCH}_{2} \mathrm{CH}_{2} \mathrm{Py}$. The
\end{abstract}


analysis of the infrared spectra made it possible to highlight, in the spectra of the complexes, new bands of vibration attributable to the axial ligands. In optical spectrometry, a new transition band, absent in the AuPc spectrum, appears around $575 \mathrm{~nm}$ in the spectra of the complexes. It is attributable to a transition with charge transfer from the central metal to the axial ligand (TC M $\rightarrow$ $\mathrm{L}_{\mathrm{ax}}$ ). Our results confirm that charge transfer transition from the axial ligand to the macrocycle (TC $\mathrm{L}_{\mathrm{ax}} \rightarrow \mathrm{Pc}$ ) are done with the assistance of the "d" orbital of the central metal; they are possible only when the electronic structure of the orbital allows it.

(C) 2018 International Formulae Group. All rights reserved.

Keywords: Gold phthalocyanine (AuPc), pyridine, substituted pyridines, vibrational spectrometry, optical spectrometry.

\section{INTRODUCTION}

Un domaine en pleine expansion en sciences des matériaux est la formation à l'échelle nanométrique, sous forme d'une phase condensée et auto-organisée, d'objets fonctionnels par assemblage de molécules organiques $\pi$-conjuguées. Les molécules organiques $\pi$-conjuguées présentent une structure favorisant des interactions intra et/ou intermoléculaires et un caractère de semiconducteur à faible gap. Elles possèdent également des propriétés optoélectroniques intéressantes que l'on peut moduler en agissant sur des groupements périphériques ou, dans le cas des complexes de coordination, en changeant l'atome métallique à leur centre (Forrest, 2004; Barth et al., 2005). D'où l'intérêt grandissant que suscitent les molécules polycycliques telles que les porphyrines et leurs homologues phtalocyanines, notamment leurs complexes de coordination (Figure 1).

Les métallophtalocyanines possèdent des propriétés particulières qui proviennent en grande partie de la forte densité électronique due à un système de dix-huit électrons $\pi$ délocalisés sur l'ensemble de la molécule. A cette forte densité électronique s'ajoute une remarquable stabilité thermique et chimique. Ces propriétés sont à l'origine de l'utilisation de ces complexes dans la fabrication des peintures et des batteries à lithium, dans la catalyse et en stockage des informations (Rawling et al., 2009; Quintiliani et al., 2005; Armstrong et al., 2000; Sakamoto et al., 2009; Al-Raqa et al., 2008; Gregory, 2000). Ils sont aussi étudiés pour des applications en photovoltaïque, en nanoélectronique, en optoélectronique et en médecine (Pandey, 2000). En outre, les complexes phtalocyanines figurent en bonne place dans la chimie supramoléculaire dont le

développement s'intéresse au stockage de l'information au niveau moléculaire, sa relecture, son transfert et sa transcription au niveau supramoléculaire (Lahmani, 2006).

La phtalocyanine d'or (AuPc) (Figure 1) qui regroupe le macrocycle phtalocyanine et le métal or pourrait avoir des propriétés remarquables (Carrascal et al., 2009). En effet, en plus des propriétés de semiconducteur organique propres à toute phtalocyanine, la présence de l'or en son centre représente un intérêt supplémentaire du fait des propriétés optique et catalytique du métal. La porphyrine d'or est utilisée comme photo-sensibilisateur dans la thérapie photodynamique du cancer et dans la catalyse; la phtalocyanine d'or étant beaucoup plus stable, ceci peut amener à la proposer comme modèle pour la conception et l'étude de nanomatériaux (Ying et al., 2005; Wieder et al., 2006). Toutefois, il existe très peu de données dans la littérature sur la phtalocyanine d'or. Jusqu'au début de ce travail, nous ne disposions que de quelques travaux sur la phtalocyanine d'or (MacCragh et al., 1965; Ouemega et al., 2011; Edwin et al., 2012; Sanda Bawa et al., 2017; Sanda Bawa et al., 2017). Ce manque d'informations a motivé le présent travail qui a pour objectif principal d'apporter une contribution à une meilleure connaissance de sa structure électronique. Dans cet article, nous présentons et discutons les résultats de la préparation et l'étude par spectrométries vibrationnelle et optique des complexes de la phtalocyanine d'or portant des ligands axiaux pyridine et pyridines substituées. Nos travaux antérieurs sur la phtalocyanine de fer (FePc) et la phtalocyanine d'or nous serviront de références. 


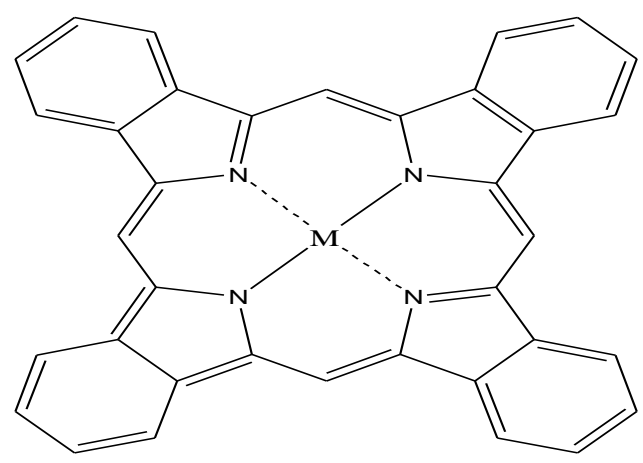

Metalloporphyrine

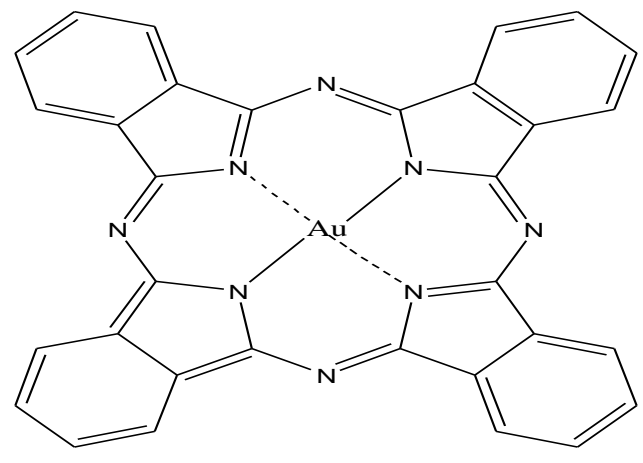

Phtalocyanine d'or

Figure 1: Représentations schématiques d'une métalloporphyrine et de la phtalocyanine d'or.

\section{MATERIELS ET METHODES} Spectrométrie vibrationnelle

Les spectres ont été enregistrés entre $400 \mathrm{~cm}^{-1}$ et $4000 \mathrm{~cm}^{-1}$ à partir de poudres des échantillons déposées sur le cristal de diamant d'un spectromètre de type Brucker TENSOR 27, ATR diamant.

\section{Spectrométrie d'absorption électronique}

Les spectres d'absorption électronique des complexes ont été enregistrés sur un spectrophotomètre SAFAS DOUBLE ENERGIE SYSTEM dans le domaine de 800 à $300 \mathrm{~nm}$. Ils ont été réalisés, à partir de solutions de diméthylformamide (DMF) et de diméthylsulfoxyde (DMSO). Ceux des complexes portant les ligands axiaux l'ont été en présence d'un excès du ligand correspondant.

\section{Préparation des composés}

Préparation de la phtalocyanine d'or (AuPc)

Nous avons préparé et purifié AuPc

conformément à un précédent travail

(Ouemega et al., 2011).

Préparations des complexes de la phtalocyanine d'or portant les ligands pyridine et pyridines substituées

Les complexes portant les ligands pyridine et pyridines substituées sont obtenus après avoir porté au reflux, dans un solvant convenable, $90 \mathrm{mg}$ de AuPc et un excès du ligand correspondant. La durée du chauffage varie avec le ligand. Elle est de trois heures avec $3 \mathrm{MePy}, 4 \mathrm{MePy}, 4 \mathrm{HOCH}_{2} \mathrm{CH}_{2} \mathrm{Py}$ et $3 \mathrm{HOCH}_{2} \mathrm{Py}$ dans l'acétonitrile; quinze heures avec $4 \mathrm{HOPy}, 3 \mathrm{HOPy}, 3 \mathrm{ClPy}$ et $4 \mathrm{ClPy}$ dans l'acétonitrile, cinq heures avec 3CHOPy, 4CHOPy, 3CNPy et une semaine avec 4CNPy dans le benzène. Les solides obtenus sont abondamment lavés à l'éthanol puis séchés.

\section{RESULTATS}

\section{Spectrométrie d'absorption infrarouge}

D'une manière générale, la fixation des ligands pyridine et pyridines substituées sur AuPc se traduit dans les spectres infrarouges par l'apparition de nouvelles bandes de vibration, la disparition et la variation des intensités relatives de certaines d'entre elles. Les fréquences de nouvelles bandes remarquables sont regroupées dans le Tableau 1.

\section{Spectrométrie d'absorption électronique.}

Le spectre de AuPc est similaire à ceux déjà publiés dans la littérature (Ouemega et al., 2011) avec deux principales bandes de transition autour de $690 \mathrm{~nm}$ (bande Q) et de $350 \mathrm{~nm}$ (bande B ou bande de Soret). Le changement de solvant n'entraîne pas de changements notables au niveau des longueurs d'onde et des intensités relatives.

Les longueurs d'onde des maxima d'absorption sont regroupées dans les Tableaux 2 et 3. Les Figures 2 à 7 présentent les spectres des complexes en solution dans le DMF ou le DMSO. 
A. S. BAWA et al. /Int. J. Biol. Chem. Sci. 12(3): 1516-1527, 2018

Tableau 1: Nouvelles bandes IR remarquables des complexes pyridine et pyridines substituées de AuPc entre 400 et $2000 \mathrm{~cm}^{-1}$.

\begin{tabular}{|c|c|c|c|c|c|c|c|c|c|c|c|c|}
\hline AuPc & - & $575 \mathrm{~F}$ & - & - & $758 \mathrm{~F}$ & & $1423 \mathrm{~F}$ & & $1608 \mathrm{f}$ & - & - & - \\
\hline $\operatorname{AuPc}(\mathbf{P y})_{2}$ & & $574 \mathrm{~F}$ & - & - & $756 \mathrm{~F}$ & & $1422 \mathrm{~F}$ & & $1606 \mathrm{f}$ & & & \\
\hline $\operatorname{AuPc}(3 \mathrm{COHPy})_{2}$ & - & $574 \mathrm{~F}$ & - & - & $756 \mathrm{~F}$ & - & $1423 \mathrm{~F}$ & - & $1607 \mathrm{f}$ & $1697 \mathrm{f}$ & - & - \\
\hline $\operatorname{AuPc}(4 \mathrm{COHPy})_{2}$ & - & $574 \mathrm{~F}$ & - & - & $755 \mathrm{~F}$ & $782 \mathrm{~F}$ & $1421 \mathrm{~F}$ & - & $1606 \mathrm{f}$ & $1703 \mathrm{f}$ & - & - \\
\hline $\operatorname{AuPc}(3 \mathrm{ClPy})_{2}$ & - & $574 \mathrm{~F}$ & & & $756 \mathrm{~F}$ & & $1422 \mathrm{~F}$ & - & $1607 \mathrm{f}$ & - & - & - \\
\hline $\operatorname{AuPc}(4 \mathrm{ClPy})_{2}$ & - & $574 \mathrm{~F}$ & & & $757 \mathrm{~m}$ & & $1420 \mathrm{~F}$ & - & $1604 \mathrm{f}$ & - & - & - \\
\hline $\operatorname{AuPc}(3 \mathrm{CNPy})_{2}$ & - & $574 \mathrm{~F}$ & - & - & $757 \mathrm{~F}$ & - & $1423 \mathrm{~F}$ & - & $1607 \mathrm{f}$ & - & $2230 \mathrm{f}$ & - \\
\hline $\operatorname{AuPc}(4 \mathrm{CNPy})_{2}$ & - & $574 \mathrm{~F}$ & - & - & $756 \mathrm{~F}$ & - & $1422 \mathrm{~F}$ & - & $1606 \mathrm{f}$ & - & $2230 \mathrm{f}$ & - \\
\hline $\operatorname{AuPc}\left(3 \mathrm{HOPy}_{2}\right.$ & - & $574 \mathrm{~F}$ & & & $756 \mathrm{~F}$ & $783 \mathrm{~F}$ & $1422 \mathrm{~F}$ & & $1606 \mathrm{f}$ & - & - & - \\
\hline $\operatorname{AuPc}(4 \mathrm{HOPy})_{2}$ & $544 \mathrm{f}$ & $574 \mathrm{~F}$ & - & - & $756 \mathrm{~F}$ & - & $1422 \mathrm{~F}$ & - & $1607 \mathrm{f}$ & - & - & - \\
\hline AuPc (3MePy & - & $574 \mathrm{~F}$ & - & - & $756 \mathrm{~F}$ & - & $1422 \mathrm{~F}$ & & $1606 \mathrm{f}$ & - & - & $2965 \mathrm{f}$ \\
\hline $\operatorname{AuPc}(4 \mathrm{MePy})_{2}$ & - & $574 \mathrm{~F}$ & - & - & $756 \mathrm{~F}$ & $782 \mathrm{~F}$ & $1421 \mathrm{~F}$ & - & $1606 \mathrm{f}$ & - & - & $2965 \mathrm{f}$ \\
\hline $\mathrm{AuPc}(3 \mathrm{HOMePy})_{2}$ & - & $573 \mathrm{~m}$ & $644 \mathrm{~m}$ & $698 \mathrm{~F}$ & $752 \mathrm{~F}$ & - & $1419 \mathrm{~F}$ & $1470 \mathrm{f}$ & $1605 \mathrm{f}$ & - & - & - \\
\hline
\end{tabular}

$\mathrm{TF}$ : Très Fort ; $\mathrm{F}$ : Fort ; $\mathrm{f}$ : faible ; tf : très faible ; $\mathrm{m}$ : moyen et $\mathrm{e}$ : épaulement. 
Tableau 2 : Longueurs d'onde des bandes de transition des complexes pyridine et pyridine substituée de AuPc en solution dans le DMF.

\begin{tabular}{|c|c|c|c|c|c|}
\hline & $\lambda_{\mathrm{e}}(\mathrm{nm})$ & $\lambda_{\mathrm{Q}}(\mathrm{nm})$ & $\lambda_{\mathrm{e}}(\mathrm{nm})$ & $\lambda_{\mathrm{e}}(\mathrm{nm})$ & $\lambda_{\mathrm{B}}(\mathrm{nm})$ \\
\hline AuPc & - & 688 & 616 & - & 351 \\
\hline $\operatorname{AuPc}(\mathrm{Py})_{2}$ & 815 & 691 & 615 & 570 & 365 \\
\hline $\mathrm{AuPc}(3 \mathrm{COHPy})_{2}$ & - & 687 & 613 & 577 & 338 \\
\hline $\mathrm{AuPc}(4 \mathrm{COHPy})_{2}$ & - & 687 & 614 & - & - \\
\hline $\mathrm{AuPc}(3 \mathrm{CNPy})_{2}$ & 820 & 687 & 613 & 575 & 371 \\
\hline $\operatorname{AuPc}(4 \mathrm{CNPy})_{2}$ & - & 687 & 614 & 580 & 360 \\
\hline $\mathrm{AuPc}\left(3 \mathrm{HOCH}_{2} \mathrm{Py}\right)_{2}$ & - & 689 & 615 & 575 & 356 \\
\hline $\operatorname{AuPc}(4 \mathrm{HOEtPy})_{2}$ & 818 & 690 & 615 & 577 & 356 \\
\hline $\operatorname{AuPc}(3 \mathrm{ClPy})_{2}$ & 820 & 687 & 614 & 577 & - \\
\hline $\mathrm{AuPc}(4 \mathrm{ClPy})_{2}$ & - & 687 & 614 & - & 362 \\
\hline $\mathrm{AuPc}(3 \mathrm{HOPy})_{2}$ & - & 687 & 614 & 577 & 354 \\
\hline $\mathrm{AuPc}(4 \mathrm{HOPy})_{2}$ & 820 & 689 & 614 & 576 & 360 \\
\hline $\mathrm{AuPc}\left(3 \mathrm{CH}_{3} \mathrm{Py}\right)_{2}$ & - & 691 & 614 & 570 & 370 \\
\hline $\mathrm{AuPc}\left(4 \mathrm{CH}_{3} \mathrm{Py}\right)_{2}$ & 820 & 691 & 614 & 575 & 362 \\
\hline
\end{tabular}

Tableau 3: Longueurs d'onde des bandes de transition des complexes pyridines et pyridine substituée de AuPc en solution dans le DMSO.

\begin{tabular}{|c|c|c|c|c|c|}
\hline & $\lambda_{Q}(\mathbf{n m})$ & $\lambda_{\mathrm{e}}(\mathrm{nm})$ & $\lambda_{\mathrm{e}}(\mathrm{nm})$ & \multicolumn{2}{|c|}{$\lambda_{\mathrm{B}}(\mathrm{nm})$} \\
\hline $\mathrm{AuPc}$ & 690 & 619 & - & & 353 \\
\hline $\mathrm{AuPc}(\mathrm{Py})_{2}$ & 692 & 620 & 576 & 365 & - \\
\hline $\mathrm{AuPc}(3 \mathrm{COHPy})_{2}$ & 687 & 616 & - & 365 & - \\
\hline $\mathrm{AuPc}(4 \mathrm{COHPy})_{2}$ & 688 & 616 & 565 & 360 & - \\
\hline $\mathrm{AuPc}(3 \mathrm{CNPy})_{2}$ & 689 & 614 & - & 367 & - \\
\hline $\mathrm{AuPc}(4 \mathrm{CNPy})_{2}$ & 688 & 614 & 560 & 360 & - \\
\hline
\end{tabular}


A. S. BAWA et al. / Int. J. Biol. Chem. Sci. 12(3): 1516-1527, 2018

\begin{tabular}{|c|c|c|c|c|c|}
\hline $\mathrm{AuPc}\left(3 \mathrm{HOCH}_{2} \mathrm{Py}\right)_{2}$ & 690 & 620 & - & 360 & - \\
\hline $\operatorname{AuPc}(4 \mathrm{HOEtPy})_{2}$ & 691 & 619 & 571 & 360 & - \\
\hline $\mathrm{AuPc}(3 \mathrm{ClPy})_{2}$ & 689 & 614 & 567 & 360 & - \\
\hline $\mathrm{AuPc}(4 \mathrm{ClPy})_{2}$ & 688 & 615 & 560 & 360 & 348 \\
\hline $\mathrm{AuPc}(3 \mathrm{HOPy})_{2}$ & 690 & 617 & $\mathbf{5 7 0}$ & 359 & - \\
\hline $\mathrm{AuPc}(4 \mathrm{HOPy})_{2}$ & 688 & 614 & 570 & 361 & - \\
\hline $\operatorname{AuPc}\left(3 \mathrm{CH}_{3} \mathrm{Py}\right)_{2}$ & 692 & 618 & 570 & 360 & - \\
\hline $\mathrm{AuPc}\left(4 \mathrm{CH}_{3} \mathrm{Py}\right)_{2}$ & 692 & 617 & 570 & 360 & - \\
\hline
\end{tabular}

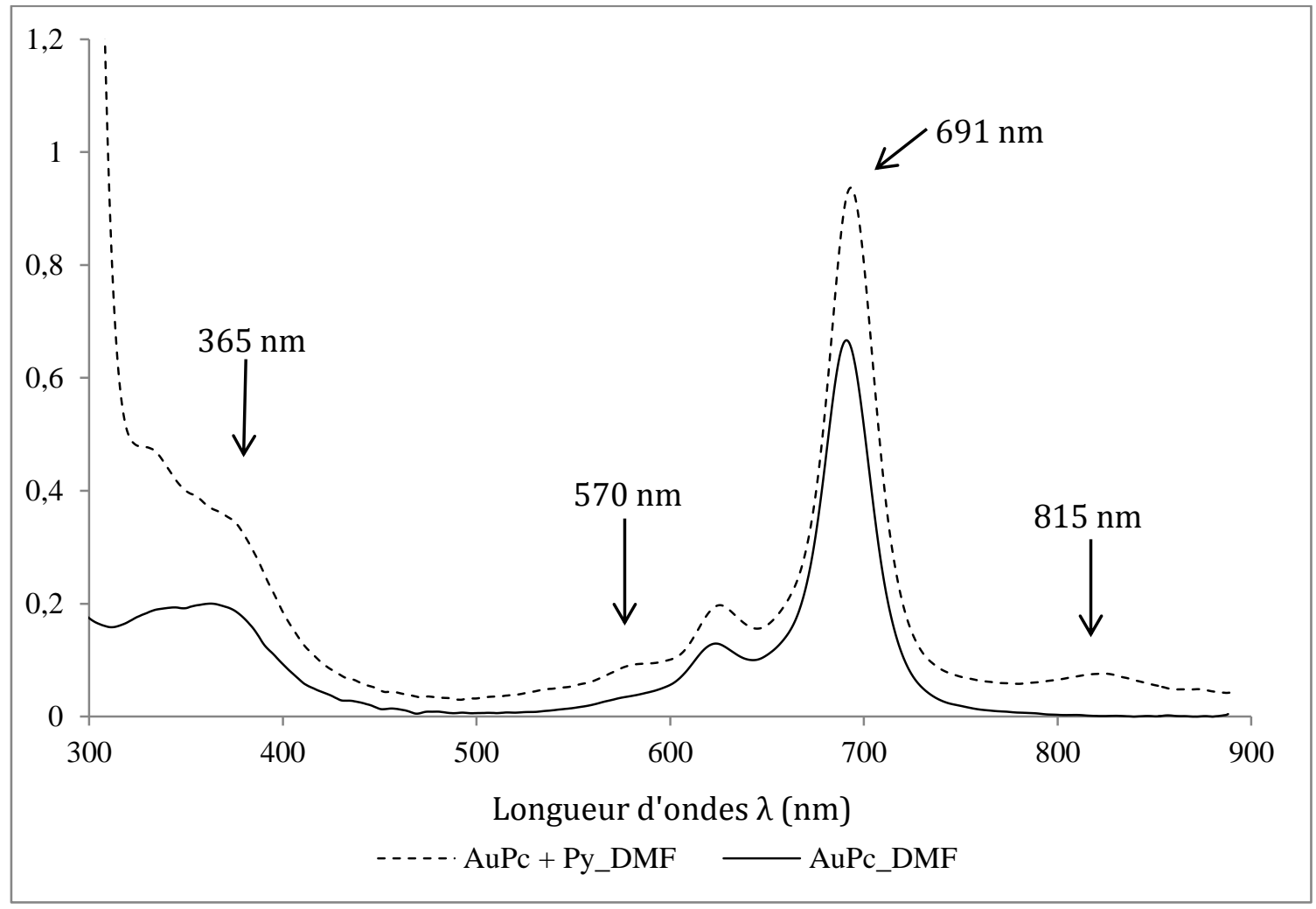

Figure 2: Spectres UV-visible de AuPc et de $\mathrm{AuPc}(\mathrm{Py})_{2}$ en solution dans le DMF. 


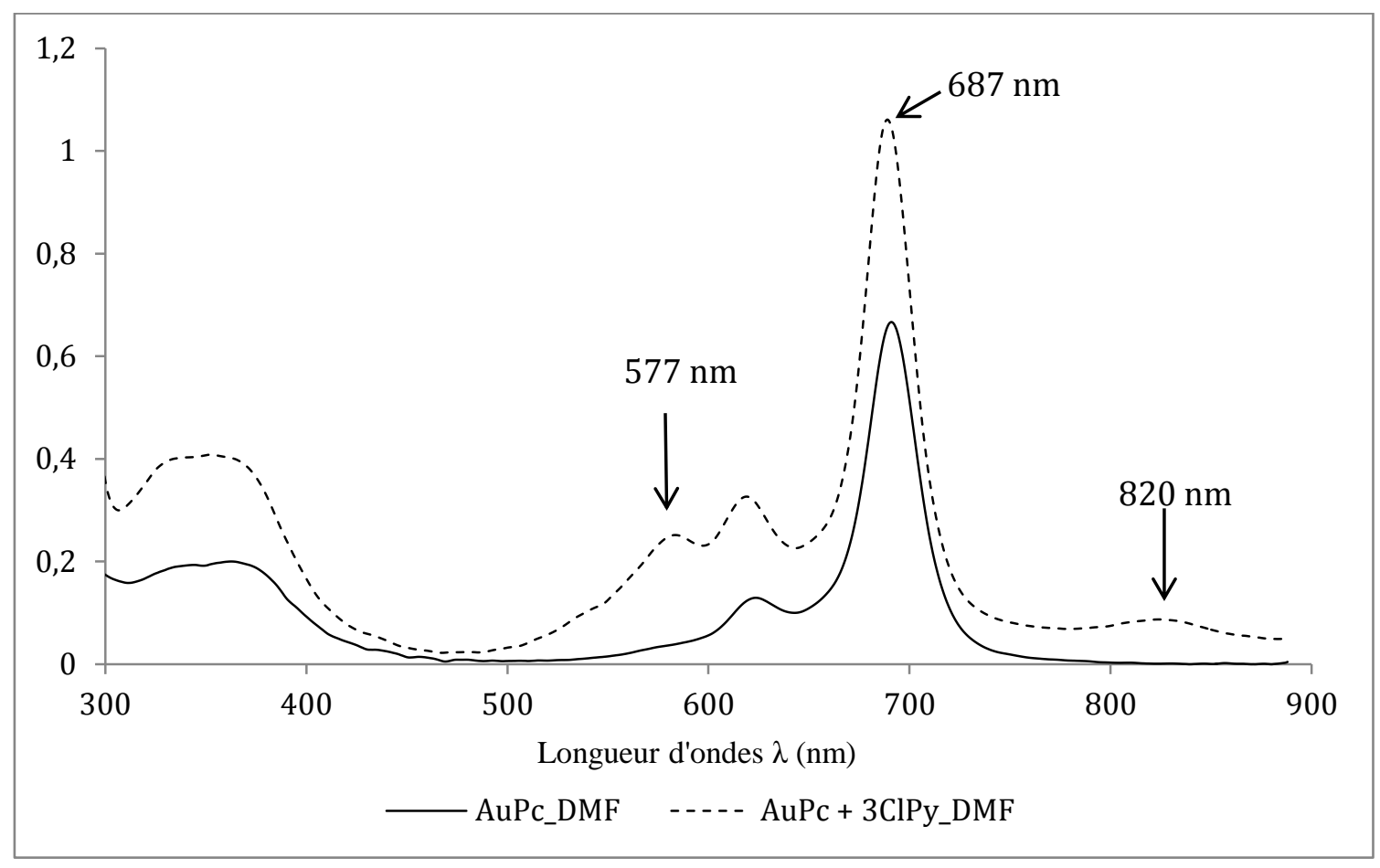

Figure 3 : Spectres UV-visible de $\mathrm{AuPc}$ et de $\mathrm{AuPc}(3 \mathrm{ClPy})_{2}$ en solution dans le DMF.

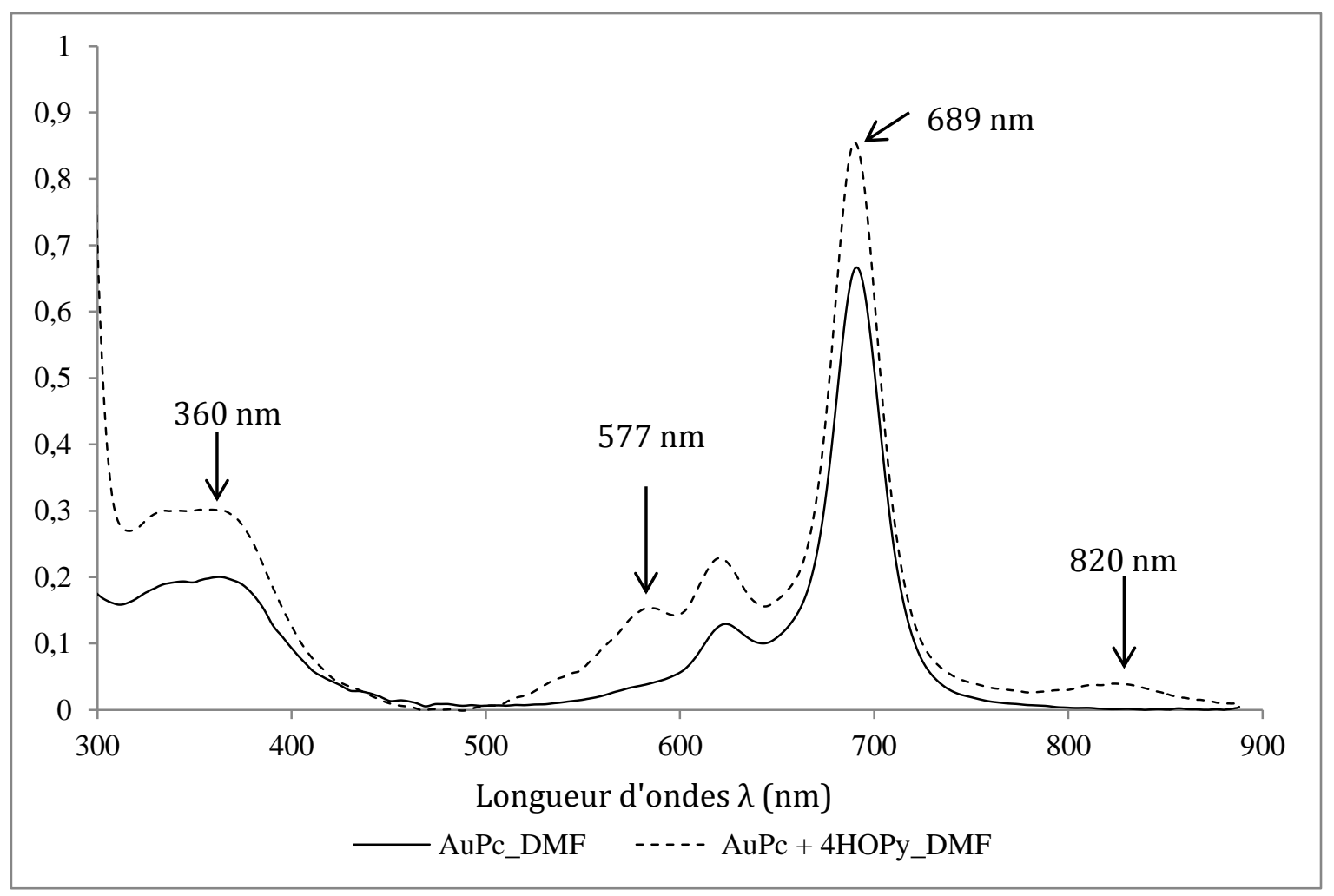

Figure 4 : Spectres UV-visible de $\mathrm{AuPc}$ et de $\mathrm{AuPc}(4 \mathrm{HOPy})_{2}$ en solution dans le DMF. 


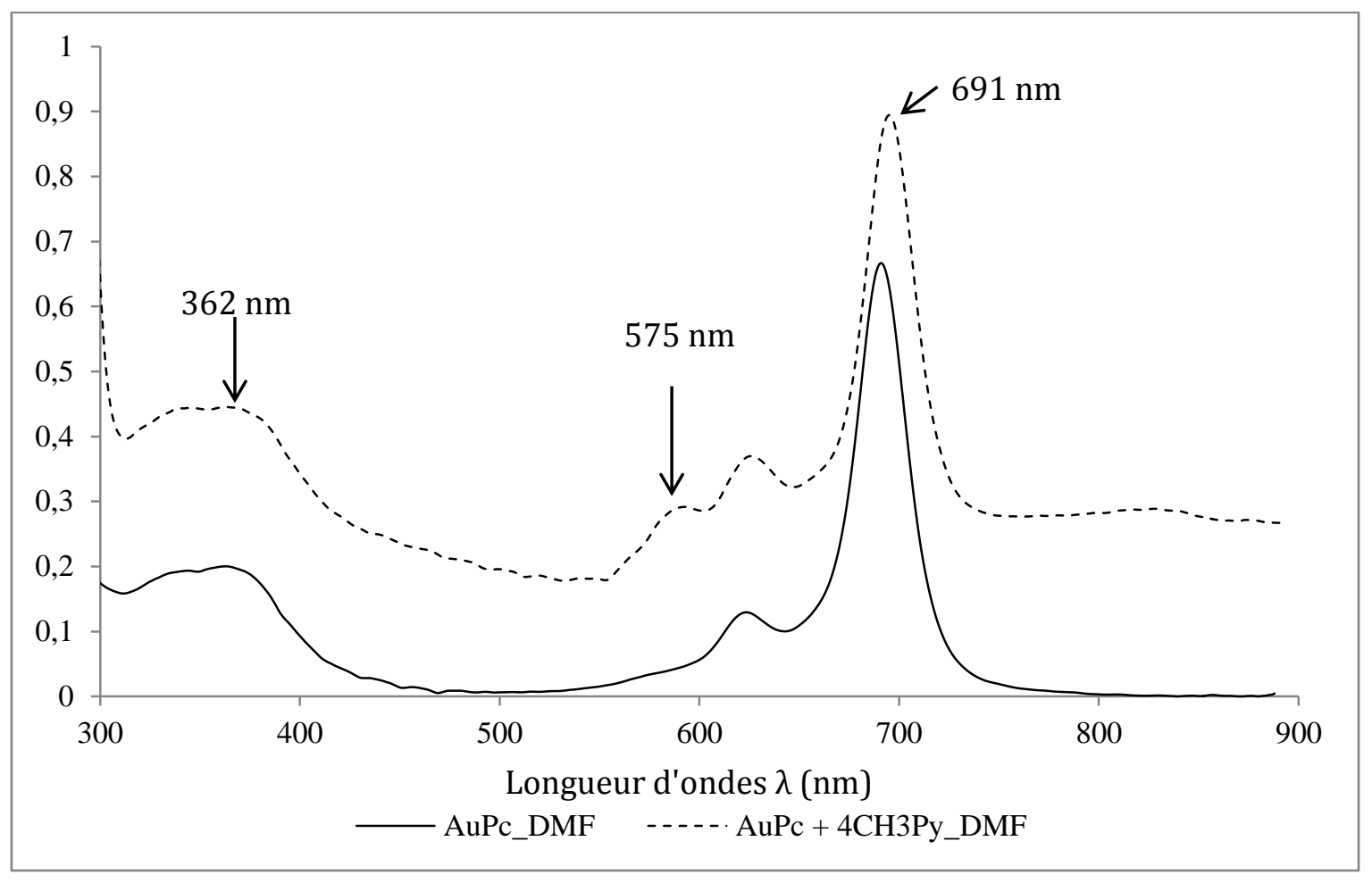

Figure 5 : Spectres UV-visible de AuPc et de $\mathrm{AuPc}\left(4 \mathrm{CH}_{3} \mathrm{Py}\right)_{2}$ en solution dans le DMF.

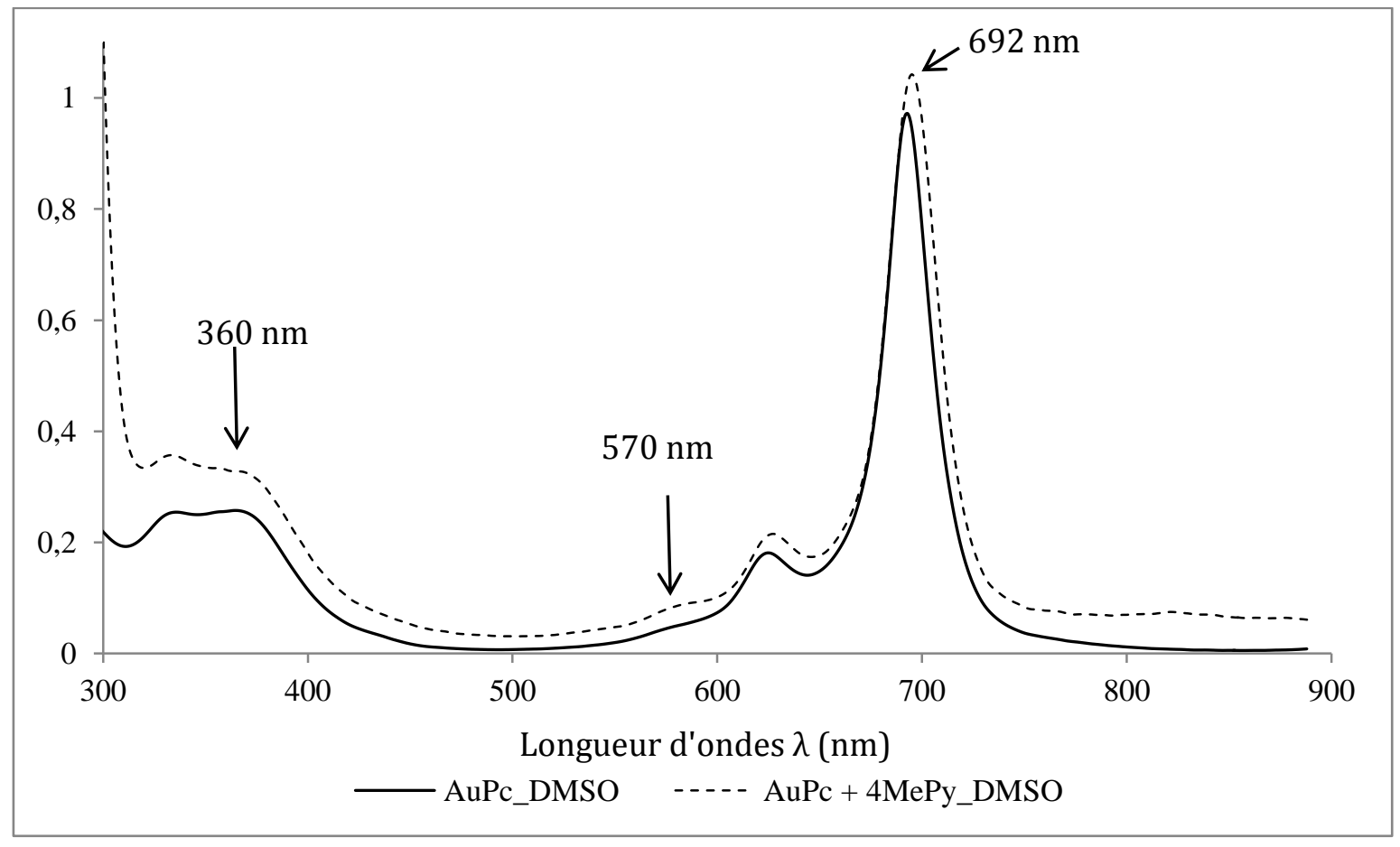

Figure 6 : Spectres UV-visible de AuPc et de $\mathrm{AuPc}\left(4 \mathrm{CH}_{3} \mathrm{Py}\right)_{2}$ en solution dans le DMSO. 


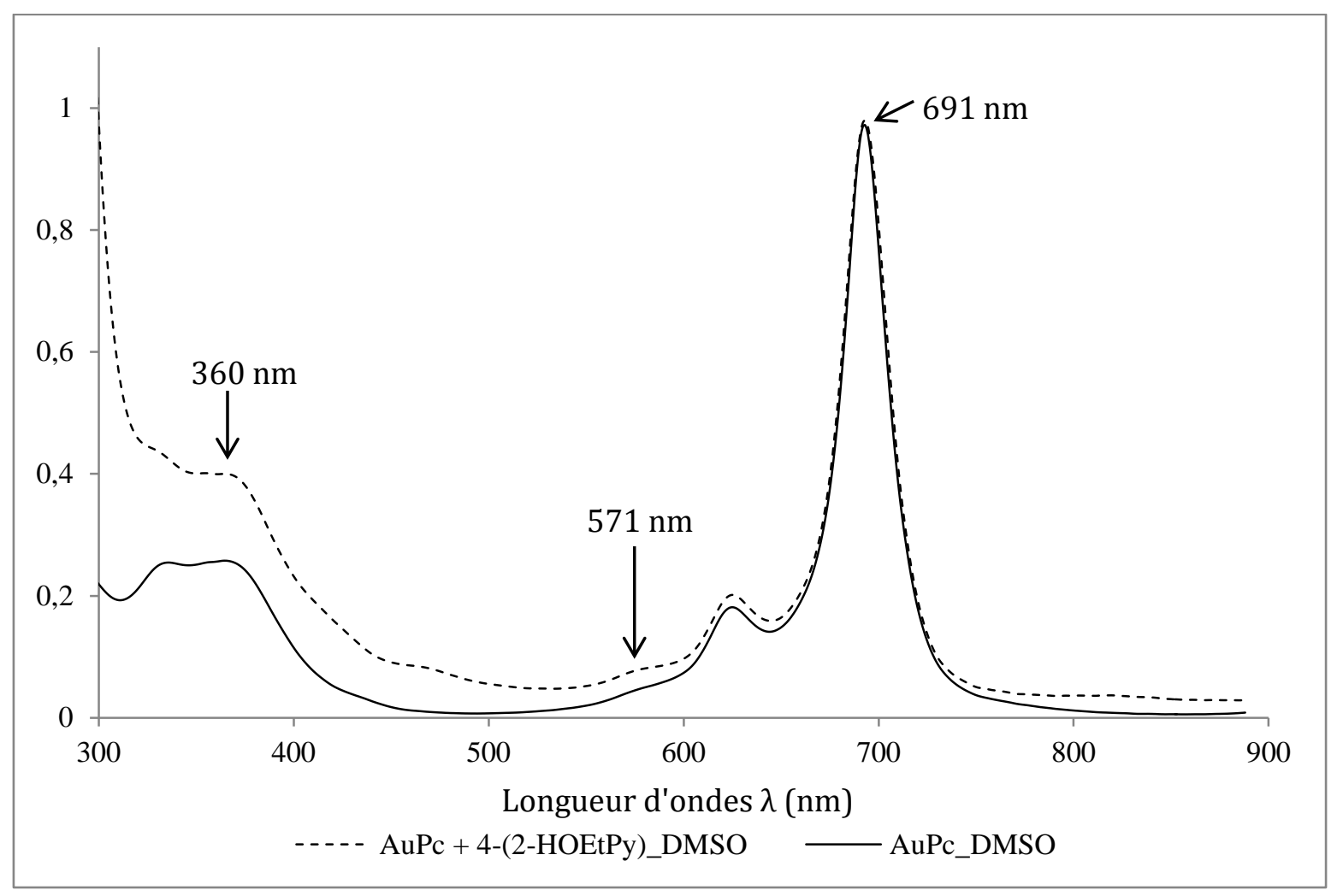

Figure 7 : Spectres UV-visible de AuPc et de AuPc[4-(2-HOEtPy) $]_{2}$ en solution dans le DMSO.

\section{DISCUSSION}

En spectrométrie d'absorption infrarouge, la bande à $544 \mathrm{~cm}^{-1}$ dans le spectre de AuPc portant le ligand 4HOPy (Tableau 1) pourrait être due à la déformation hors du plan de la liaison $\mathrm{O}-\mathrm{H}$ par analogie avec celle observée dans le spectre de la molécule de phénol (Laure, 2005). Celle à $1470 \mathrm{~cm}^{-1}$ dans le complexe portant le ligand $3 \mathrm{HOCH}_{2} \mathrm{Py}$ (Tableau 1) proviendrait du cisaillement de l'entité $-\mathrm{CH}_{2^{-}}$par comparaison avec celle obtenue à $1471 \mathrm{~cm}^{-1}$ dans le spectre de l'alcool benzylique (Laure, 2005). Les bandes à $699 \mathrm{~cm}^{-1}$ et $644 \mathrm{~cm}^{-1}$ dans le spectre du composé de réaction de $\mathrm{AuPc}$ avec le ligand $3 \mathrm{HOCH}_{2} \mathrm{Py}$ (Tableau 1) peuvent être attribuées aux vibrations $\mathrm{C}-\mathrm{H}$ de la pyridine. Ces deux bandes de vibration sont présentes à $703 \mathrm{~cm}^{-1}$ et $653 \mathrm{~cm}^{-1}$ dans le spectre de la pyridine libre (Kabayashi, 2005). Les bandes de faibles intensités à $1697 \mathrm{~cm}^{-1}$ et $1703 \mathrm{~cm}^{-1}$, respectivement dans les spectres des composés de réaction de $\mathrm{AuPc}$ avec les ligands 3COHPy et 4COHPy (Tableau 1), peuvent être attribuées aux vibrations de la liaison $\mathrm{C}=\mathrm{O}$ de la pyridine substituée (Siham, 2012).

L'exploitation du domaine de fréquences $1800-4000 \mathrm{~cm}^{-1}$ nous a permis de mettre en évidence d'autres bandes de vibration des groupements fonctionnels des ligands axiaux (-CN, $-\mathrm{CH}_{3}$ etc..) que nous avons utilisés. Ainsi, dans les spectres des composés de réaction de AuPc avec 3CNPy et 4CNPy (Tableau 1), on observe une bande de vibration de faible intensité à $2230 \mathrm{~cm}^{-1}$ attribuable à $v_{(\mathrm{CN})}$. Cette bande a été observée à $2221 \mathrm{~cm}^{-1}$ dans les composés de la pyridine (Siham, 2012). Dans la littérature, les bandes de vibration du groupement méthyl de la picoline $\left(\mathrm{CH}_{3} \mathrm{Py}\right)$ apparaissent entre 2900 et $3000 \mathrm{~cm}^{-1}$ (Varsanyi, 1969). Elles sont observées à $2957 \mathrm{~cm}^{-1}$ dans le spectre de la 2picoline (2- $\left.\mathrm{CH}_{3} \mathrm{Py}\right)$ (Arenas, 1997), et 
respectivement à $2957 \mathrm{~cm}^{-1}$ et à $2924 \mathrm{~cm}^{-1}$ dans ceux de la 3-picoline (3- $\left.\mathrm{CH}_{3} \mathrm{Py}\right)$ et de la 4-picoline (4- $\left.\mathrm{CH}_{3} \mathrm{Py}\right)$ (Lopez, 1998). Par analogie, nous attribuons la bande autour de $2965 \mathrm{~cm}^{-1}$ dans les spectres des composés de réaction de AuPc avec les ligands $3 \mathrm{CH}_{3} \mathrm{Py}$ et $4 \mathrm{CH}_{3} \mathrm{Py}$ (Tableau 1) aux vibrations du groupement $-\mathrm{CH}_{3}$. L'observation de nouvelles bandes de vibration attribuables aux ligands axiaux confirme la réaction de $\mathrm{AuPc}$ avec les différents ligands utilisés.

Dans l'ensemble, les fréquences des bandes caractéristiques du macrocycle phtalocyanine $(\mathrm{Pc})$ sont peu modifiées en dépit de la fixation des ligands axiaux. Ceci montre la grande stabilité des liaisons $\mathrm{C}-\mathrm{C}, \mathrm{C}$ $\mathrm{N}$ et $\mathrm{C}=\mathrm{N}$ du macrocycle phtalocyanine. L'invariance des bandes de vibrations associées aux différents mouvements du métal dans le plan du macrocycle phtalocyanine, malgré la substitution en position axiale, permet de voir que la symétrie autour du métal n'est pas modifiée (Sanda, 2018). Ces constats militent en faveur de la formation de complexes hexacoordinés $\mathrm{AuPc}(\mathrm{RPy})_{2}$.

En spectrométrie d'absorption électronique, les spectres des composés de réaction de $\mathrm{AuPc}$ avec les ligands présentent les bandes caractéristiques de AuPc et une nouvelle bande de transition autour de $575 \mathrm{~nm}$ dans le DMF (Figures 2-5) et autour de 570 nm dans le DMSO (Figures 6-7).

Des travaux déjà effectués sur des complexes de $\mathrm{FePc}$ portant les ligands phosphines et phosphites ont permis de différencier les complexes hexacoordinés des complexes pentacoordinés en spectrométrie optique. Les spectres des complexes pentacoordinées $\left(\mathrm{FePcPX}_{3}\right)$ dans le DMSO présentent une bande $\mathrm{Q}$ dédoublée (Zanguina, 2002). Ces mêmes caractéristiques spectrales ont été observées avec des composés tels que $\mathrm{FePcCl}, \mathrm{FePcSR}, \mathrm{FePcH}_{2} \mathrm{O}$ et FePcPy (BayoBangoura et al., 2003). Alors que les spectres des complexes hexacoordinés présentent une bande Q non dédoublée (Zanguina, 2002). Les spectres des composés de réaction de AuPc avec les ligands pyridine et pyridines substituées ne présentent pas une bande Q dédoublée. Nous pouvons en déduire que les composés obtenus sont des complexes hexacoordinés.

Les travaux antérieurs sur les composés hexacoordinés obtenus par réaction de $\mathrm{FePc}$ avec les ligands pyridines et pyridines substituées, mettent en évidence deux bandes de transition avec transfert de charges (Ouédrago et al., 1981; Bayo et al., 1992). L'une autour de $415 \mathrm{~nm}$ attribuée à un transfert de charges $\mathrm{L}_{\mathrm{ax}} \rightarrow \mathrm{Pc}$ et l'autre autour de $550 \mathrm{~nm}$ attribuée à une transition avec transfert de charges $\mathrm{Fe} \rightarrow \mathrm{L}_{\mathrm{ax}}$. Nous attribuons donc la nouvelle bande de transition autour de $575 \mathrm{~nm}$ dans le DMF et autour de $570 \mathrm{~nm}$ dans le DMSO à un transfert de charges $\mathrm{Au} \rightarrow$ $\mathrm{L}_{\mathrm{ax}}$.

La bande de transition avec transfert de charges $\mathrm{Fe} \rightarrow \mathrm{L}_{\mathrm{ax}}$ n'avait été observée que lorsque le substituant sur la pyridine était électro-attracteur. Ces résultats avaient permis de conclure que cette transition est liée à la nature du ligand axial et des substituants équatoriaux (Bayo, 1989). Dans le présent travail, elle est présente dans les spectres de tous les composés de réaction de AuPc avec les ligands axiaux; elle est alors indépendante de la nature du ligand axial. Ce nouveau résultat nous permet de dire que si elle peut dépendre de la nature du ligand axial et des substituants équatoriaux, elle est aussi étroitement liée à la nature du métal central.

L'absence d'une seconde bande de transition attribuable à une transition avec transfert de charges $L_{a x} \rightarrow$ Pc pourrait être liée au fait que l'or ne dispose probablement pas d'orbitale $« \mathrm{~d} »$ favorisant une telle transition. Ce qui confirme que cette transition se fait avec l'assistance des orbitales « $\mathrm{d}$ » du métal central (Ouédraogo et al., 1981).

\section{Conclusion}

Ce travail a permis de préparer et d'isoler, sous forme solide, pour la première fois, une série de complexes de AuPc portant les ligands axiaux: Py, 3MePy, 4MePy, 3HOPy, 4HOPy, 3ClPy, 4ClPy, 3СOHPy, 4COHPy, 3CNPy, 4CNPy, $3 \mathrm{HOCH}_{2} \mathrm{Py}$ et $4 \mathrm{HOCH}_{2} \mathrm{CH}_{2} \mathrm{Py}$. La présence, dans les spectres des complexes portant les ligands axiaux, de nouvelles bandes de vibration attribuables aux vibrations des ligands axiaux 
confirme que les réactions attendues ont été réalisées. La coordination des différents types de ligands axiaux est confirmée par la spectrométrie UV-visible à travers l'apparition d'une nouvelle bande de transition avec transfert de charges $\mathrm{Au} \rightarrow \mathrm{L}_{\mathrm{ax}}$. L'analyse des résultats de spectrométrie optique conduit à une structure hexacoordinée des complexes étudiés : AuPc[RPy $]_{2}$.

Nous prévoyons mettre à profit les propriétés de ces complexes pour former des molécules hybrides par conjugaison de ces complexes avec des nanoparticules.

\section{CONFLIT D'INTERETS}

Les auteurs déclarent qu'ils n'ont aucun conflit d'intérêt.

\section{CONTRIBUTION DES AUTEURS}

ASB est le principal investigateur; BO a initié les travaux sur la phtalocyanine d'or dans notre laboratoire; MB-B a dirigé les travaux; KB a supervisé les travaux.

\section{RÉFÉRENCES}

Al-Raqa SY. 2008. The synthesis and photophysical properties of novel, symmetrical, hexadecasubstituted $\mathrm{Zn}$ phthalocyanines and related unsymmetrical derivatives. Dyes and Pigm., 77: 259265. https://doi.org/10.1142/S108842460 6000077.

Arenas JF, Lopez Town I, Otero JC, Marcos JI. 1997. Vibrational spectrum of 2methylpyridine. Journal of Molecular Structure., $\quad$ 410(411): $\quad 443-446$. https://doi.org/10.1016/S00222860(96)09699-8.

Armstrong NR. 2000. Phthalocyanines and porphyrins as materials. J. Porphyrins Phthalocyanines, 4: 414-417. DOI: 10. 10021 (SICI) 10991409(200006/07)4:4<414::AIDJPP247>3.0.CO;2-B.

Barth JV, Constanyini G et Kern K. 2005. Engineenig atomic and molécular nanostructure at surfaces. Nature, 171: 437. DOI: $10.1038 /$ nature04166.

Bayo-Bangoura M, Bayo K, Ouédrago GV, Mossayan JC, Terzian G, Benlian D.
2003. Preéparation et caractérisation par Rayons X, par spectrométries UV-visble, IR, Mössbauer et RPE de composés thiolates de la phtalocyanine de fer. $J$. Ouest-Afr. Chem., 16: 77-96.

Bayo K. 1989. Contribution à l'étude des transferts de charges dans les composés de la ferrophtalocyanine. Thèse de $3^{\text {ème }}$ cycle. Université de Ouagadougou, Burkina Faso, 118 p.

Bayo K, Saba A, Ouédraogo GV, Terzian G, Benlian D. 1992. Dérivées substituées de la ferrophtalocyanine. J. Mol. Struct., 271: 19-26. DOI: https://doi.org/10.1016/00222860(92)80206-W.

Carrascal D, Fernandez-Seivane L et Ferrer J. 2009. Oscillating spin density pattern in gold metallocen an phthalocyanine molecules. Phisical review B., 80: $184415 . \quad$ DOI: https://doi.org/10.1103/PhysRevB.80 .184415 .

Edwin YW, Akito M, Daniel BLeznoff. 2012. Gold(II) Phthalocyanine Revisited: Synthesis and Spectroscopic Properties of Gold(III) Phthalocyanine and an Unprecedented Ring-Contracted Phthalocyanine Analogue. Chem. Eur. J., 18: 12404-12410. DOI: $10.1002 /$ chem. 201201701 .

Forrest S. 2004. The path to ubiquitous an low cost organic electronic appiances on plastic. Nature., 911: 428. doi: 10.1038/nature 02498 .

Gregory P. 2000. Industrial applications of phthalocyamines. J. Porphyrins Phthalocyanines., $\quad 4$ : 368-373. https://doi.org/10.1002/(SICI)10991409(200006/07)4:4<432::AIDJPP254>3.0.CO;2-N.

Kabayashi T, Furokawa F and Uyeda N. 1970. Spectrochim. Acta., 26A: 1035.

Lahmani M, Bréchignac C, Houdy P. 2006. Les Nanosciences. 2. Nanomatériaux et nanochimie : Belin.

Laure Emmanuël. 2005. Identification Spectrométrique de Composés Organiques (traduction de la $5^{\text {eme }}$ édition américaine). DeBoeck Université : Paris; $1998 \mathrm{p}$. 
Lopez Town I, Woolley MS, Otero JC, Marcos JI. 1998. Vibrational spectrum of 3-methyl and 4-methylpyridine. Journal of Molecular Structure., 470: 241-246. https://doi.org/10.1016/S00222860(98)00376-7Get rights.

MacCragh A, Koski WS. 1965. The phtalocyanine of gold. J. Am. Chem. Soc., $\quad 87$ : 2496-2497. DOI: $10.1021 / \mathrm{ja} 01089 \mathrm{a} 038$.

Ouédraogo S. 2011. Etude des interactions intramoléculaires dans les bimétallophtalocyanines de zinc et de cobalt par des méthodes électrochimiques. Thèse de Doctcrat Unique. Université de Ouagadougou, Burkina Faso, 69 p.

Ouemega B, Bayo-Bangoura M, Bayo K. 2011. Préparation et étude de la phtalocyanine d'or. J. Soc. Ouest-Afr. Chim., 031: 42-48. www.soachim.org/files/volume_31/5B.\%20Ouemega\%20et\%20al.pdf.

Pandey RK. 2000. Recent advances in photodynamic therapy. J. Porphyrins Phthalocyanines, 4: 368-373. https://doi.org/10.1002/(SICI)10991409(200006/07)4:4<368::AIDJPP244>3.0.CO;2-6.

Quintiliani M, Garcia-Frutos EM, Gouloumis A, Vásquez P, Ledoux-Rak I, Zyss J, Claessens CG, Torres T. 2005. Synthesis, Characterisation and nonlinear optical properties of twodimensional octupolar systems based on phthalocyanine compounds. Eur. J. Org. Chem., 18: 3911-3915. DOI: $10.1021 /$ jo991843f.

Rawling T, Austin C, Buchholz F, Colbran SB. 2009. Ruthenium phthalocyaninebipyridyl dyads as sensitizers for dyesensitized solar cells: Dye coverage versus molecular efficiency. Inorg. Chem., 48: 3215-3227. DOI: $10.1021 / \mathrm{ic} 802087 \mathrm{n}$.

Sakamoto K, Ohno-okumura E. 2009. Syntheses and functional properties of phthalocyanines. Materials, 2: 11271179. Doi:10.3390/ma2031127.

Sanda BA, Tarpaga L, Bayo-Bangoura M, Ouédraogo S, Bayo K. 2017. Préparation et études vibrationnelle et électronique de complexes de la phtalocyanine d'or avec des ligands axiaux phosphines et phosphites. J. Soc. Ouest-Afr. Chim., 43: 11-16.

http://www.soachim.org/files/volume_43 $12 \% 20$ AS\%20Bawa\%20et\%20al,\%20Vo 1043,\%20(2017)\%2011-17\%20bon.pdf.

Sanda BA, Bayo-Bangoura M, Ouédraogo S, Tarpaga L, Bayo K. 2017. Préparation et études vibrationnelle et électronique de complexes de la phtalocyanine d'or avec des ligands axiaux thiolates. J. Soc. Ouest-Afr. Chim., 44: 43-50. http://www.soachim.org/files/volume_44 17-

AS\%20Bawa\%20et\%20al\%20Vol\%200 44\%20(2017)\%20\%2043-50.pdf.

Sanda BA. 2018. Préparation et études par spectrométries vibrationnelle et électronique de complexes de la phtalocyanine d'or avec des ligands axiaux phosphines, phosphites, thiolates, pyridine et dérivés de pyridine. Thèse de Doctcrat Unique. Université de Ouaga I Pr Joseph KI-ZERBO, Burkina Faso, 67 p.

Siham AbdulRahman Al-Issa. 2012. Synthesis of a New Series of Pyridine and Fused Pyridine Derivatives. Molecules., 17: 10902-10915.

Doi: 10.3390/molecules170910902.

Varsanyi G. 1969. Vibrational Spectra of Benzene Derivatives. Academic Press: New York.

Wieder ME, Hone DC, Cook MJ, Handsley MM, Gavrilovic J and Russell DA. 2006. Photochem. Photobiol. Sci., 5: 727. DOI: 10.1039/b602830f.

Ying Wang, Qing-Yu He, WaiYin Sun R, Chi-Ming Che, and Jen-Fu Chiu. 2005. Cancer res., 65(24): 11553-11564. DOI: 10.1158/0008-5472.CAN-052867 Published December.

Zanguina A. 2002. IR and UV-visible spectra of iron (ii) phthalocyanine complexes with phosphine or phosphite. Bull. Chem. Soc. Ethiop., 16(1): 73-79. https://www.ajol.info/index.php/bcse/arti cle/viewFile/20950/3913. 